\title{
The Futuristic Microchip Drug Delivery System - A New Identification Tool
}

\author{
Ranajit Nath ${ }^{1}$, Ratna Roy ${ }^{2}$, Jisu Das ${ }^{3}$, Kritideepa Nath ${ }^{1}$, Priyanka Kurmi ${ }^{1}$, Ambika Mandal ${ }^{1}$ \\ ${ }^{1}$ Department of Pharmaceutics, NSHM Knowledge Campus, Kolkata- Group of Institutions, Kolkata, West \\ Bengal, India \\ 2Department of Pharmacology, NSHM Knowledge Campus, Kolkata- Group of Institutions, Kolkata, West \\ Bengal, India \\ ${ }^{3}$ Department of Pharmaceutical Sciences , Dibrugarh University, Dibrugarh, Assam, India
}

\begin{abstract}
Article Info

Volume 8, Issue 4

Page Number : 59-73

\section{Publication Issue}

July-August-2021

\section{Article History}

Accepted : 01 July 2021

Published : 05 July 2021

The microchip medication delivery system is the most incredible technique to administer medicine over a lengthy period of time without requiring the patient's intervention. It is made up of a number of drug-filled sockets (typically 50-300) that release medication at specified intervals. A large number of reservoirs may be built on a single microchip via micro fabrication. The substance to be administered is injected into the reservoir. The reservoir can hold a variety of chemicals or other compounds in various dosages. In recent years, several studies have been performed to enhance medicine delivery productivity and to find a dependable method for medication distribution inside the body. Despite the fact that the controlled release system is the most significant drug delivery technology, micro drug delivery methods for hormones, vaccines, and anticancer medicines have seen a rise in attention. This article basically seeks information regarding the mechanism of action of microchip, its clinical and preclinical studies and its applications in the delivery of the drugs.
\end{abstract}

Keywords : Microchips, Technology, Medical Science

\section{INTRODUCTION}

The microchip medication delivery system is the most amazing way to distribute a drug for an extended period of time without the need for the patient to intervene. It comprises of a variety of drug-filled sockets (usually ranging from 50 to 300 ) that release the medicine at predetermined intervals. Microchips' primary technique for drug delivery is based on hermetically sealing small amounts of drug in micro- reservoirs and releasing that drug on demand or on schedule. There are several fundamental issues in medication delivery: Storage and protection of the chemical for a long time appropriate distribution (i.e., timing and pharmacokinetics), specific amounts of a chemical released at predetermined intervals, adherence to the specified treatment.

A microchip system can store a huge number of medications or chemicals, control when the chemicals 
are released, and control the pace at which they are released. A drug delivery device can distribute a wide range of medications in a regulated, pulsatile, or continuous manner, implanted within the human body. The microchip might be connected to a small power supply and controlled by a computer. Biosensors, a microprocessor, or a remote control.[1]]

We created simple microchips for drug release based on diffusion through micro channels to enable longterm medication delivery. The microchips were made of poly(methyl methacrylate) and included one or more micro-wells and controlled-length micro channels. To act as a drug diffusion barrier, the channels were filled with a biocompatible polymer called poly(ethylene glycol). The springs packed with a fine powder of a model molecule, fluorescein, functioned as drug reservoirs. Three different drug delivery microchip designs were created, each having a 1,4 , or $8 \mathrm{~mm}$ channel length. [2]

Synthesis or discovery of novel chemical compounds used to be a big deal back in the day. Entities having desired therapeutic characteristics but no pharmacological qualities. One of the main goals of the study was to eliminate unfavourable side effects research into pharmaceuticals. The pace and breadth of medication absorption have been disclosed in pharmacokinetic and pharmacological research. Therapeutics are ultimately regulated by absorption rather than dosage in the treatment of systemic diseases (Urquhart et al., 1984). This has resulted in the creation of drug delivery systems that are effective. Pharmacological selectivity that isn't exclusively based on chemical properties not just on the basis of structure, but also on the basis of a drug's regulated rate providence (Goldman 1982; Banerjee \& Robinson, 1991). [3]

Using traditional methods, drug release profiles were established (oral dose forms, aerosols, injections, etc.) A medication concentration is usually included in creams, lotions, and ointments surge immediately after administration, followed by a drop in medication concentration (linear or parabolic) with the passage of time. This type of release profile will suffice in most cases when the therapeutic medication concentration is high. With time, the range is enormous or almost constant. However, there are numerous illnesses in which it is present . It would be desirable, and in certain situations necessary, if control the release of drugs more accurately. [4]

In recent years, there has been a greater emphasis on improving drug delivery efficiency. It is expected that the demand for drugs would increase. All throughout the world, delivery methods will improve world. Despite the fact that the controlled release system in the single most powerful medication delivery system. There has been a surge in interest in this method for the delivery of tiny medication delivery systems. Hormones, anticancer drugs, and vaccines are only a few examples. Rapid advances in medicine necessitate rapid responses. Modifications to the medication delivery mechanism Lower adverse effects, drug delivery effectiveness, and ease of use patient care, cheaper costs, and ease of usage. The highest focus is given to comfort.[5]

\section{MECHANISM OF ACTION}

It is one of the first completely MEMS-based medication delivery systems . This delivery system comprises of a substrate with numerous reservoirs capable of storing compounds in solid, liquefied, or gel form FIG1.The final circuitry is connected into each reservoir, which is capped with a conductive membrane. A microprocessor is in charge of this . By regulating the breakdown of the gold membrane, the central processor must be able to regulate the exact moment of release and amount of medicines distributed electrically. Multiple sealed compartments are included in the design, which are opened on demand to give medication doses. The prime step in making these microchips was to use a vertical tube 
reactor to deposit of low stress siliconnitride on both sides of prime grade,(100) silicon wafers.[6]

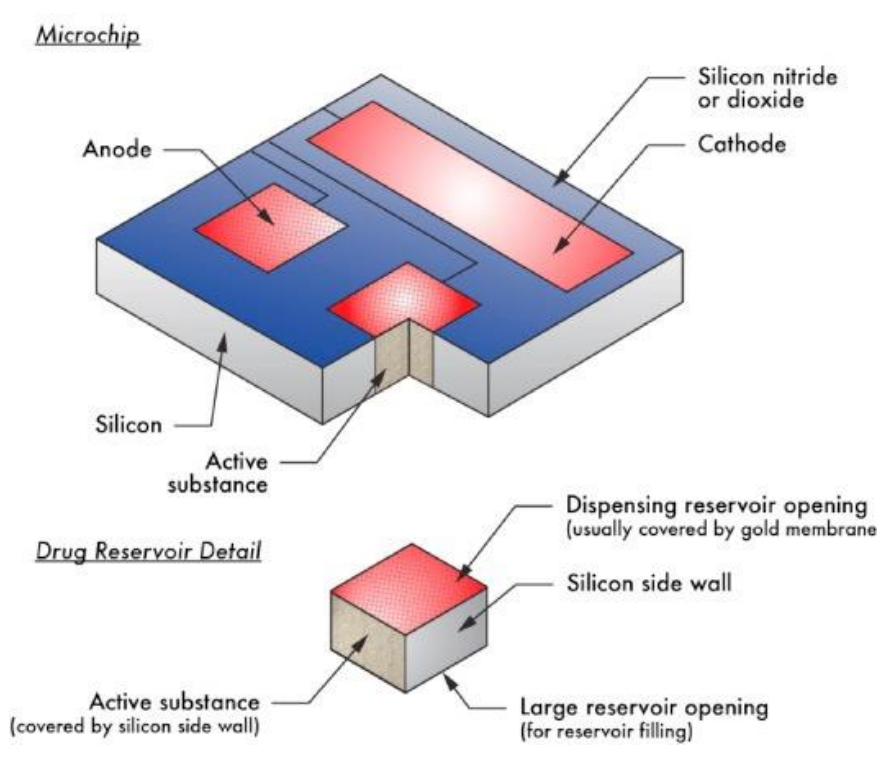

Fig 1: A Typical Controlled Release Microchip[7.]

The chip's operation is relatively simple. Electrocapillarity and dielectrophoresis are used to operate electrically controlled droplet-based labs-ona-chip. Manipulation of electrified droplets requires sophisticated micro fluidic mechanics[8]. Under the unified framework of droplet electro hydrodynamics, we investigate these operational principles, particularly electro wetting on dielectric (a kind of electrocapillarity) and dielectrophoresis. We distinguish them based on their electric sources and energy transmission methods. Both electro wetting on dielectric and dielectrophoresis are useful for droplet production and manipulation, according to the findings. The findings demonstrated simulations of electrically driven droplet formation, translocation, fusion, and fission. An electrical potential of is induced, resulting in the formation of a soluble gold chloride complex. If the anode potential continues at this level, gold dissolution can proceed. The dissolution of this thin gold barrier allows the medication to enter the circulation and reach its intended destination in the body.[9,10].



Fig - 2 : Mechanism of Action of Microchip

\section{PRECLINICAL TRIALS}

Drug development is an expensive and timeconsuming procedure. According to a 2012 research, drug development costs $\$ 1.2$ billion each drug that is successfully developed. Also, it takes an average of 10-15 years for a new drug to reach the market after extensive preclinical and clinical testing. Only $27 \%$ of drugs that make it to the clinical trial phase of the drug development process are authorized for commercialization. Lack of efficacy (43\%) and toxicity (33\%) are the two most common causes for drugs failing in clinical trials ${ }^{[1]}$.

\section{Early Health Technology Assessment}

Early HTA, which includes early health economic modelling, is becoming more popular as an approach for determining the added value of potential new technologies early in the development process. Such evaluations would be important: 
1. In deciding on the technology's future development.

2. To establish minimal performance thresholds for the new technology in comparison to existing technologies.

3. To assist in the early phases of development with price and reimbursement ${ }^{[1]}$.

Early HTA can assist developers in making design decisions for devices while they are still in development. In a period where the developers can still make substantial changes to the design, a

systematic assessment can still be conducted. As a result, developers may enhance their chances of developing a successful product that will be accepted by end-users and regulatory bodies by doing early HTA $^{[1]}$.

In early HTA, a variety of approaches can be employed, including surveys to assess potential impact, economic modelling to analyze costs and benefits, and scenario-driven computer simulations of clinical trials. Because it may be used to integrate the opinions of all stakeholders, using multicriteria decision analysis (MCDA) in early HTA can be a valuable tool in the decision-making process ${ }^{[1]}$.

Preclinical drug testing models that are predictive of drug response in human tissues or organs are urgently needed to reduce the required financial and time investment in the development of new pharmaceutical agents ${ }^{[2]}$.

The use of animal models in drug development and evaluation is regarded as a critical tool in medicine for researching new therapies and evaluating new drugs. Despite the widespread use of these models, drug success rates in human clinical trials have remained significantly low. The substantial evolutionary differences between human and animal models, which result in higher structural and biological complexity of human tissues or organs, can be attributed for this level of failure ${ }^{[2]}$.

The use of genetically engineered animals, particularly mice, is one way to address such problems. E.g., The established distinction between the expression patterns of the CYP2B gene in mice and humans is that in mice, many functional members of the CYP2B family are expressed, but in humans, only CYP2B6 is the only functional member ${ }^{[2]}$.

Drug metabolization by UDP-glucuronosyltransferase (UGT) enzymes is another species difference investigated in this manner. In 2010, UGT1-null mice were crossed with human UGT1 transgenic mice to create humanized UGT1 mice, according to a recent review of toxicity and metabolic research in hUGT1 mice $^{[2]}$.

Human and mouse artificial chromosome vectors, as well as chimeric mice with humanized livers, are other examples ${ }^{[2]}$.

Preclinical and clinical research account for 33\% and $63 \%$, respectively, of the total cost of drug development. Only one novel chemical entity (NCE) out of 10,000 receives regulatory clearance. Target identification is the first step toward approval, and it entails identifying a specific potential target (cell receptors, proteins, enzymes, DNA, RNA, and ribosomal proteins, among others) that might be responsible for the disease. Once identified, the target must be validated using experimental data by assessing the effects on certain pathologies, molecular and physiological inhibition, as well as modulation activities that support the target's potential therapeutic goals. Computer simulations may be used to predict the biologic effects and toxicity of chemical or drug candidates, narrowing down the possibilities to the most promising molecules to be tested in vivo. Computer aided drug design, for example, can be used to anticipate receptor binding sites for a potential drug molecule. Quantitative $S A R$ can assess the link between physicochemical properties and biological activity, and structure-activity relationship software can predict the biologic activity of a drug candidate. Furthermore, computer software may be used to evaluate and forecast the carcinogenicity and mutagenicity of drug candidates. Cost-effectiveness, 
viability of producing the lead drug, and drug delivery methods are all assessed at this stage ${ }^{[2]}$.

\section{Pharmacokinetics (PK) and pharmacodynamics (PD)} studies are the two primary concerns throughout the preclinical stage of drug development. The fate of a drug administered is determined by PK during the five phases of absorption, distribution, metabolism, excretion, and toxicity (ADMET) PD studies look at how a drug and its products affect the body's physiology in order to evaluate efficacy, toxicity, and dose. To imply that these research might be safe to advance to human clinical trials, they must be conducted in at least two distinct species. In vitro micro-physiological platforms capable of determining the ADMET of drug candidates during preclinical stages might help speed up the drug development process $^{[2]}$.

Aside from using common disease cells, several animal models were utilized to study drug metabolism and therapeutic efficacy in relation to a target model. Because of its short lifespan of 2-3 weeks, Caenorhabditis elegans (C. elegans) is an excellent model for ageing and metabolic research. Researchers might investigate the impact of drugs on C. elegans development and describe drug metabolism. Similarly, for embryonic research, the zebra fish modelintegrated microfluidic device was utilized to monitor dynamic drug effects ${ }^{[3]}$.

\section{Evaluation of drug toxicity}

The evaluation of drug toxicity is necessary for the safe development of new pharmaceuticals and is usually done using animal and cell-based models. However, in vivo tests have several drawbacks, including ethical concerns, high costs, and the inability to conduct quantitative studies or high throughput assays, all of which limit their use. The fast advancement of organ-on-chip technology may give a viable option for simulating the complex physical environment of the human body in vitro ${ }^{[3]}$.
Organ chips have also been utilized in drug toxicity research in recent years. Drug absorption-related nephrotoxicity may be efficiently assessed using an intestine-kidney-on-a-chip with multi-interfaces and compartmentalized micro-chambers. A kidney-on-achip capable of mimicking the nephron was also utilized to explore the pathophysiology of druginduced acute renal damage and to offer drug-induced nephrotoxicity evaluations ${ }^{[3]}$.

Microfluidic chips can also be used to evaluate the toxicity of drug compounds. For example, in order to evaluate cardiac toxicity, researchers used a highthroughput, dependable, and less expensive platform to examine the growth and culture properties of HEK cells ${ }^{[3]}$.

Recent advances in microfluidic cell cultures enable the construction of in vitro human skin models that can be used for drug toxicity testing and disease study. A 'skin-on-a-chip' approach was developed to mimic the structures and functional responses of the human skin to evaluate the effects of therapeutic drug testing models. Recent developments in microfluidic cell cultures have made it possible to create in vitro human skin models for drug toxicity testing and disease research. To analyze the impacts of therapeutic drug testing models, a "skin-on-a-chip" approach was developed to replicate the structures and functional responses of human skin ${ }^{[3]}$.

Organ-on-a-chip technology, which reflects the genetic features of cells in each patient, has recently attracted interest as a potential tool for drug development and clinical drug testing. By mimicking the cellular behavior of the target tissue milieu, OOC is an engineered assembly of a controlled compartment that may be used to study, measure, and regulate cell behavior and responsiveness to various drug stimuli. To overcome the limitations of traditional drug testing models, OOC has emerged from a combination of diverse engineering platforms such as microfluidic systems, engineered biomimetic tissues, and non-invasive monitoring systems. It is now possible to design organoids with two- 
dimensional (2D) and three-dimensional (3D) scaffolds equipped with appropriate extracellular matrix (ECM) to accurately replicate human cell adhesion, migration, differentiation, and function in vitro due to rapid advancements in engineered biomaterials. Organoids for the OOCs were created using a variety of human and animal stem cells. Personalized tissue constructs or disease models can be engineered using human-induced pluripotent stem cells (hiPSCs), which can be derived from a patient's skin tissue or directly harvested as pathogenic cells from a patient. As a result, hiPSC-integrated OOCs may be used to create personalized drug testing platforms that imitate human physiology and are tailored to certain patient groups and individuals. The use of disease-specific cell lines, primary cells, and hiPSCs has substantially eliminated restrictions such as the availability of patient-specific human cells, which used to limit the potential of OOCs only a decade ago. OOCs are a useful technique for capturing complex drug interactions within multiple organ systems due to the unlimited renewability and potency of hiPSCs to differentiate into main cell types and generate diverse types of tissues or organoids ${ }^{[2]}$.

To conduct sophisticated fluid manipulation and enable for repeated measurements and labor-free testing, the OOC is paired with a micro-device-based non-invasive monitoring component (e.g., sensors and miniaturized microscopes) and an automated microfluidic control system ${ }^{[2]}$.

Organs-on-chips are biomimetic micro-engineered systems that mimic the essential functioning units of living human organs. They are typically made up of transparent 3D polymeric microchannels lined with living human cells that mimic three key aspects of intact organs: 3D microarchitecture defined by the spatial distribution of multiple tissue types, functional tissue-tissue interfaces, and complex organ-specific mechanical and biochemical microenvironments. These systems might be utilized as specialized in vitro models that provide simulation, mechanistic investigation and pharmacological modulation of complicated biological processes ${ }^{[4]}$.

This biomimetic microsystems method has been utilised to create micro-engineered models of human organs such as the liver, heart, lung, gut, kidney, brain, and bone that replicate their structural and functional complexity ${ }^{[4]}$.

Organs-on-chips have the potential to be a novel enabling platform for identifying and validating the efficacy, safety, and druggability of potential targets early in the pipeline, improving the chances of clinical trial success. Song et al. ${ }^{[5]}$ utilized a microengineered model of vasculature to mechanistically examine chemokine-mediated interactions between circulating breast cancer cells and the micro vascular endothelium, which is an excellent example of such an application. ${ }^{[4]}$.


Fig - 3 : Cancer research using organ-on-a-chip $\operatorname{models}^{[4]}$

a) A compartmentalized microfluidic device enabled basal stimulation and activation of endothelial cells cultured on a porous membrane using chemokines to examine the attachment of circulating breast tumor cells implicated in cancer metastasis using a micro vascular endotheliumon-a-chip. By introducing chemokines such tumor necrosis factor to the bottom channel, the effect of these agents was studied. The 
endothelium that had been pre-treated with tumor necrosis factor had more cancer cells adhering to it than the endothelium that had not been treated ${ }^{[4]}$.

b) b) Human umbilical vein endothelial cells were grown in a microfluidic channel adjacent to a $3 \mathrm{D}$ collagen gel containing bone cells differentiated from human bone marrow-derived mesenchymal stem cells (MSCs) to study breast cancer cell metastasis to bone. The cancer cells were seen migrating into the bone ${ }^{[4]}$.

c) Lung cancer spheroids were embedded in micropatterned 3D matrices immediately adjacent to a microchannel lined with endothelial cells to examine epithelial-mesenchymal transition in cancer. Micro-fluorometry is used to detect dispersion of cancer spheroids during epithelialmesenchymal transition analysis. PMDS i.e., Poly (dimethylsiloxane).

ORGAN-ON-CHIP TECHNOLOGY is being used to develop cost-effective in vitro models for hitto-lead and lead optimization, as well as innovative phenotypic screening assays, that can more reliably predict the efficacy, toxicity, and pharmacokinetics of drug compounds in humans ${ }^{[4]}$.

In clinical studies, poor efficacy is caused by the absence of predicted therapeutic effects or the unexpected need to increase the dose to levels where toxicity is also apparent. In many situations, the unfavorable outcome is due to existing methods' failure to properly predict in vivo drug efficacy prior to clinical trials. Human organ-on-a-chip models are ideally adapted to address the limitations of present techniques by allowing researchers to reproduce and pharmacologically alter key aetiologies and clinically important integrated downstream responses at various degrees of complexity ${ }^{[4]}$.

$\checkmark$ A recent research developed a micro-engineered 3D assay of epithelial-mesenchymal transition (EMT) during cancer progression, as shown in fig 1c, to examine the potential of organ-on-chip approaches for testing drug efficacy ${ }^{[4]}$.

Unexpected adverse drug effects are the second most prevalent reason of clinical trial failures, and they are also the cause of costly drug withdrawals. Established biochemical models can evaluate specific and predicted toxicity mechanisms (e.g., hERG (also known as KCNH2) channel binding; the hERG is a potassium voltage-gated channel), whereas cellular models may monitor simplistic end points as cytotoxicity. Animal testing often misses important human toxicity, resulting in the early rejection of drug candidates based on animal-specific toxicity pathways.

Organ-on-chip technology might be used to evaluate human-relevant drug responses for expected toxicities at multiple levels of biological complexity (subcellular, cellular, tissue, and organ levels), as well as to detect unforeseen off-target toxicities $^{[4]}$. As a result, organ-on-a-chip models may be utilized for precise prediction and mechanistic research of dose-limiting human toxicities of potential medicines, as well as the discovery of new treatment methods to reduce the reported toxic effects. Predictions made by these models in the drug discovery pipeline might inform and facilitate early attempts to identify, modify, and optimize lead compounds, resulting in safer drugs with a higher chance of success in clinical trials ${ }^{[4]}$.

Several studies have shown that organ-on-chip micro-devices may be used to simulate key pharmacokinetic processes that regulate drug bioavailability, with a focus on drug metabolism. Liver-on-a-chip models combined with physiologically based pharmacokinetic computational models have shown to be particularly effective in predicting metabolic drug clearance rates that are consistent with clinical evidence $^{[4]}$. 
$\checkmark$ Phenotypic screening: Target-based drug discovery necessitates the development and testing of specific mechanistic molecular hypotheses in order to identify therapeutic targets and drug candidates, whereas phenotypic drug discovery is fueled by the screening of specific physiological responses in cell-based or animal models. The objective of phenotypic screening is to identify active compounds that ameliorate disease phenotypes without having to worry about the compounds' molecular mechanisms of action $^{[4]}$.

$\checkmark \quad$ It is important that the assay captures different elements of complicated human physiology while being accessible to high-throughput manipulation for efficient appraisal of large compound libraries for successful phenotypic screening ${ }^{[4]}$.

$\checkmark \quad$ In addition, phenotypic screening can be used to repurpose approved drugs or drugs that failed to fulfil their primary efficacy end points in Phase III clinical trials for the treatment of other diseases. Serendipitous observations in animal research, clinical trials, and clinical practice have led to the majority of successfully repositioned drugs, all of which are costly. As a result, phenotypic screening utilizing predictive preclinical models has been proposed as the most efficient and cost-effective method of repositioning drugs ${ }^{[4]}$.

Organ-on-chip systems might be beneficial in supporting and accelerating efforts in rare diseases, stratified medicine, and nanomedicine, sectors that are gaining growing industry interest, in addition to enhancing research and development efficiency in general. Integrating organs-on-chips with other emerging disease modelling techniques, such as sourcing cells from patients to develop induced pluripotent stem cells (iPS cells) or modifying normal stem cells with new gene editing tools (e.g., the clustered regularly interspaced short palindromic repeats
CRISPR) - Cas system), could make it easier to investigate these new applications ${ }^{[4]}$.

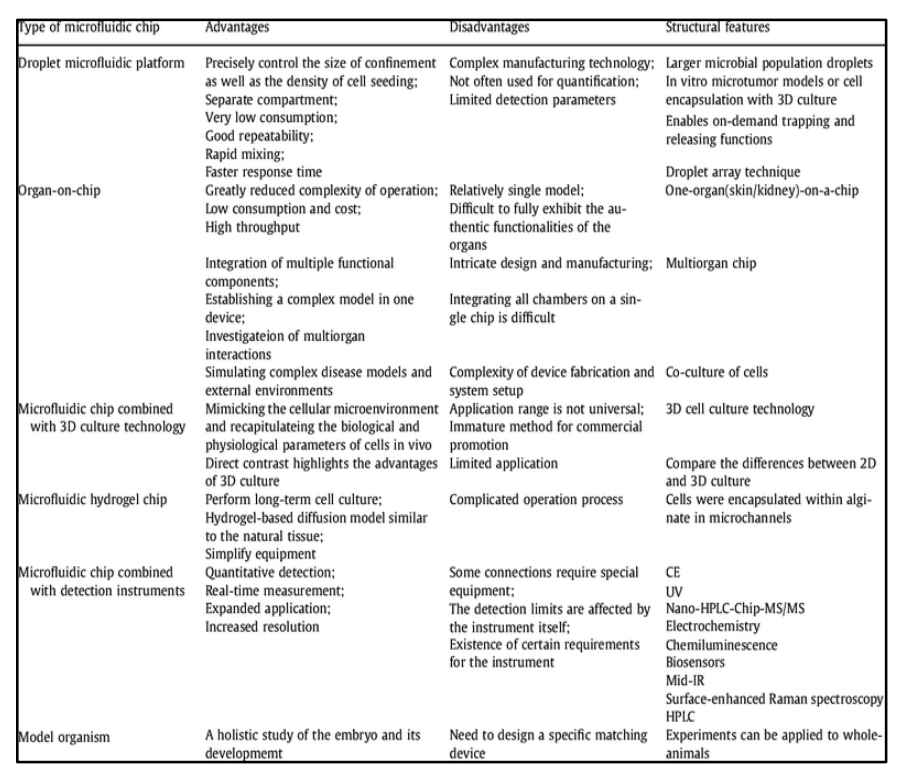

Fig 4 : Microfluidic Application In Pharmaceutical Analysis ${ }^{[3]}$ :

\section{CLINICAL TRIALS ON MICROCHIP}

Many people suffer from life-threatening diseases that are difficult to treat, such as cancers, neurological disorders, and pediatric and rare diseases. Clinical trials are an important strategy for these people in order to identify effective new and repurposed therapies. Despite promising preclinical test findings in conventional models, such as $2 \mathrm{D}$ cell culture systems, and animal models, such as mice, around $85 \%$ of late-stage clinical trials of experimental medicines fail due to safety issues or ineffectiveness.

Microchips have been utilized in human research (e.g. dialysis) and can be used to treat a variety of diseases, including diabetes and hypertension. Furthermore, the use of microchips has been observed in the creation of artificial glands. The significant role of hormone regulation in the body, which is directly linked to dysfunctional glands, in both regulating existing disease states and preventing them via other hormonally induced diseases ${ }^{[6]}$. 
Organ-on-a-chip technology has been created by scientists as an alternative to animal testing in order to enhance assay validity and throughput capabilities (3D human living cell cultures that are cultivated in a dynamic microchip environment under controlled condition that maintain human tissue functionality or mimic organ dysfunction). As a result, these diseasespecific human cell types may be utilized to create individual micro tissues with physiologic cellular behavior, and organ-on-a-chip technology can be utilized for disease modelling in vitro. In this regard, various chemicals or drugs are incorporated into microchips (solid silicon based), which are commonly used in medical implants ${ }^{[6]}$.

Professor Kevin Warwick achieved the first demonstration of microchip implantation in a human for identifying and tracking purposes in 1998[6].

Micro CHIPS received the 2008 AAPS Drug Delivery Technology Award for its osteoporosis research in November. The American Association of Pharmaceutical Scientists gives the award to recognize exceptional research in the field of novel drug delivery technologies The Micro CHIPS device is being developed to deliver human parathyroid hormone (hPTH 1-34) to assist patients with osteoporosis grow bone, prevent future fractures, and enhance their quality of life.

A survey of 150 compounds resulting in human toxicity events during clinical development summarized and compared drug toxicity in humans and animal models and determined major deficits as rodent-only models were predictive of human toxicity in $43 \%$ and non-rodent only were predictive of $63 \%$, with a combined true positive human toxicity concordance rate of $71 \%$. As a result, the intricate nature of human diseases makes it difficult for animal models to fully replicate them, and the expected response and recovery might differ dramatically from the triggered reaction in humans. Vioxx for rheumatoid arthritis, TGN1412 for immunotherapy, the HIV vaccine, and Parkinson's disease therapies
CEP-1347 and Cogane are just a few instances of failed studies ${ }^{[2]}$.

- There is currently just one registered clinical trial that intends to gather human samples for the purpose of developing biomimetic human OOC technologies for disease modelling and researching the function of the micro-biome in the pathogenesis of human gastrointestinal diseases. However, no registered clinical trials employing the OOC for drug screening or validating the use of OOC technologies for this purpose are currently available. Problems in phase 4 clinical trials, post-marketing failures, and black-box warnings (i.e., strictest warning in the labeling of drugs and products given by the Food and Drug Administration) are all critical problems in the pharmaceutical industry ${ }^{[2]}$.

\section{APPLICATIONS OF MICROCHIP}

\section{DNA chips to aid in the diagnosis of brain tumours}

Because of their heterogeneity and variable malignancy, current methods for treating brain tumours are difficult and challenging. Gliomas are the most common brain tumours found in adults, and their diagnosis is based on subtle microscopic characteristics. There is currently no distinguishing marker or genetic signature that can predict the outcome of each type of glioma. A group of researchers from the Institute Curie and Inserm used DNA chip technology to identify tumours with the best prognosis, whose chromosome had undergone a specific deletion. Comparative Genomic Hybridization (CGH) is one of these analytic methods, which allows for a global assessment of the genome. It's a tool for reconfiguring genome regions that have been amplified or deleted, which happens a lot in tumour cells.[16] 


\section{In Cancer Therapy}

Doctors use protein levels in the blood to determine a patient's cancer risk and to assess the health of elderly people with chronic diseases. However, the current methods for testing these proteins require far too much blood to be performed on a regular basis and are also prohibitively expensive. The researchers hoped to make bedside diagnostics based on blood proteins a reality by lowering the cost of such tests. [16]

\section{Microchip for Antidepressants}

Depression is the world's fourth leading cause of disability. Mechanical devices like the microprocessor-based Medication Event Monitoring System (MEMS) have recently been developed. The blood assay for drug and its metabolites has also been used for dothiepin; a ratio of nordothiepin:dothiepin greater than 1.1 indicates noncompliance for a period of 48 hours or longer. The MEMS system has made it possible for us to detect the exact opening times of the reservoirs. The great advantage of implantable systems in chronic depression is that patients are liberated from taking the medicines for months or years.[16]

\section{Microfluidic cell treatment}

Micro-fluidic devices enable the manipulation of individual objects of cell size and therefore the analysis can be obtained under controlled yet physiological environments. Furthermore, by parallelizing applied methodologies, a large number of cells can be observed at the same time and under similar conditions.[16]

\section{Simplicity of release mechanism}

Chemicals are liberated from the microchip as a result of membrane fragmentation. The membrane is shattered when an electric potential is applied, causing the membrane to dissolve via a simple electrochemical reaction. There are no movable segments on the microchip device. The lack of moving parts may improve device reliability by reducing the possibility of mechanical breakdown.[16]

\section{Accuracy of dose}

The microchip device has multiple reservoirs, and each reservoir of the microchip can be precisely filled with a small amount of the drug using the microinjection or ink-jet printing method. It is critical that the amount of drug administered to a patient matches the amount prescribed, especially for highly potent compounds. The amount of drug administered from a microchip filled using this printing technique can be tightly controlled, and accidental overdose can be avoided because active devices can only be released when an electric potential is applied to an anode. Larger doses can be delivered by simply opening multiple reservoirs at the same time.[16]

\section{Improve shelf-life}

Several protein-based drugs have a short shelf life or stability. One of the most common causes in this case is water permeation into these protein drug formulations. The membrane layering a microchip's packed reservoir will prevent water from diffusing into these reservoirs. Thus, the stability of a protein drug can be improved by first isolating it from the outside environment (hermetically sealing it) and then storing the microchip in its most stable form (solid, liquid or gel).[16]

\section{Potential for local delivery}

The microchip device can be made infinitesimal to enable local chemical delivery. The main advantage of local drug delivery is that high concentrations of drug can be achieved at the site of application while keeping systemic concentrations low. For example, BCNU (carmustine) is widely used in the treatment of malignant brain tumours. A large amount of BCNU must be administered to a patient in a systematic manner in order to achieve minimal adequate concentrations at the tumour site in the brain, which causes damage to the liver, spleen, and kidney. The 
use of polymer wafers containing BCNU at the tumour site allows for a 1000 -fold increase in local BCNU concentrations over systemic delivery while maintaining systemic concentrations low.[16]

\section{Complex release patterns}

The microchip can be used to obtain the concomitant constant and pulsatile release (Complex release patterns). Any complicated chemical or drug release pattern can be broken down into two parameters: release time and release rate. Controlling both of these parameters is a distinguishing feature of a controlled release microchip.[16]

\section{Compounds to be released}

Many substances can be placed inside the microchip's reservoir unit and released from the microchip in due course. Each reservoir can be filled with a unique chemical or chemical mix. Chemicals that are filled into the reservoir can be solid, liquid, or gel. The delivery of liquids is restricted to microfluidic devices such as pumps.[16]

\section{Current Applications: \\ Recent Studies and Patents}

A review of recent microchip advances, important patents, and clinically relevant applications can provide insight into the current state of microchips in medicine while also stimulating ideas for additional research. Santini Jr. et al. were issued the US Patent "Microchip Drug Delivery Devices" in 1998, which was the first to describe the characteristics of a multireservoir microchip system with an active release system. The first electrochemically triggered drug delivery microchip was introduced by Santini Jr. et al. in 1999. Release from individually dosed reservoirs is activated in their device by applying an electric potential between the cathode and the anode- a thin gold membrane covering the specific reservoir to be deployed. Since then the group has discovered and patented a large number of further advances, including (not just) refined manufacturing procedures, microchip flexibility and improved flexibility, operating methods and wireless data details and power transfers.[17]

\section{Brain Cancer}

The first study of polymer microchip in vivo efficacy for brain cancer therapy demonstrated that microchips, when combined with the appropriate application and therapeutic agents, could be clinically implemented. Applications in chronic illnesses such as brain cancer, where continuous and controlled local drug administration to a difficult-to-access anatomical site is sought, show that microchip technology has a bright future.[17]

\section{Osteopenia and Osteoporosis}

Farra et al. studied the human in vivo pharmacokinetics of $\mathrm{hPTH}(1-34)$ delivered via microchip devices in eight female patients with osteopenia or osteoporosis in 2012. To allow for the creation of a tissue capsule, the devices were triggered 8 weeks after implantation. The pharmacokinetic characteristics of the released parathyroid hormone were found to be day-to-day repeatable by device and bioequivalent to injections of FORSTEO, a commercially available hPTH(1-34) therapy. Total biocompatibility, safety, and patient satisfaction were also documented, as were biomarkers of skeletal response and bone development.[17]

\section{Abdominal Implantation}

The creation of a tissue capsule around the microchip device, which could modify the bioactive agent's release kinetics and reduce efficacy, is a worry of using microchips for medication release. The researchers demonstrated that the release kinetics were comparable to injections using serum samples. The researchers exposed the microchip (and tissue capsule) to histological testing after removing it from the abdominal cavity, taking into account the 
implant's potential for adverse consequences or immunological reactions (photo of microchip and tissue capsule is shown in Figure 1 \& 2). Six of the seven capsules showed typical wound healing responses and normal inflammatory cell levels. The seventh capsule histology sample revealed an increased number of macrophages, but they were still within normal limits. These findings added to the growing body of evidence supporting the use of microchips in people.[17]

In conclusion, the study's biggest flaw was discovered to be overall equipment functionality. One of the eight implanted devices did not release any drug and thus was excluded from the study. Despite this flaw, this seminal study demonstrated the practical and effective use of microchips in medicine as an alternative to multiple regular injections.[17]

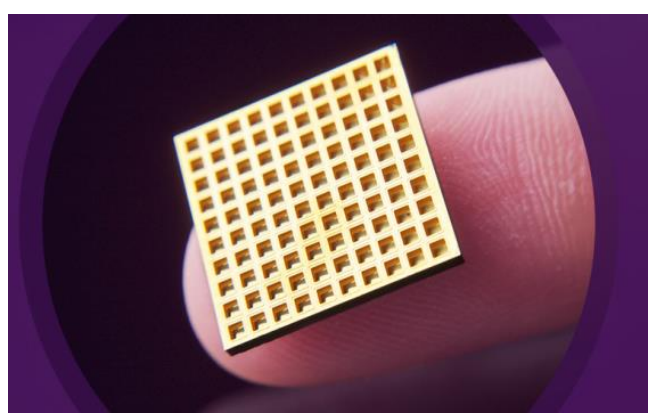

Fig1: Microchip[18]

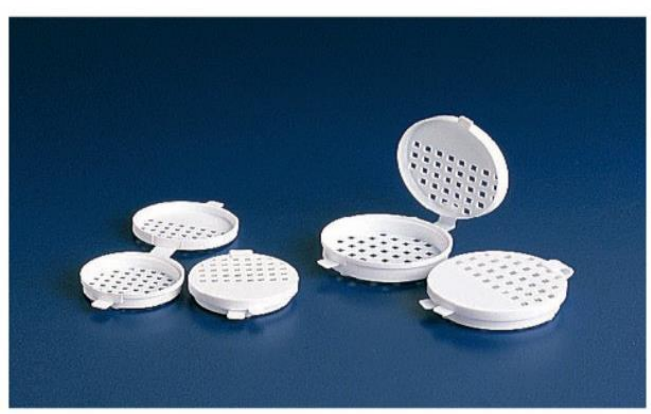

Fig2: Tissue Capsule[19]

\section{Future Applications}

The widespread use of microchip technology has the potential to revolutionise the modern healthcare system. Therapeutic processes will be altered, billions of dollars in unnecessary costs will be avoided, and patient populations' quality of life will improve.[17]

Treatments for diseases like diabetes and hypertension that require dose titrations could be changed to provide safer and more effective automated therapy regimens. This controlled-release technique, when used in conjunction with implants, reduces the risk of foreign body reactions and rejection, as well as inflammation and pain, allowing the body to heal more quickly following surgery. Microchip applications could be expanded to build artificial glands. Hormone regulation in the body linked to malfunctioning glands could help control current illness states as well as prevent the advent of other hormone-related illnesses.[17]

Microchip delivery systems will help in the treatment of diseases with a low rate of compliance (mental disorders, some cancer medicines, long-term antibiotics, etc.) or abuse potential. Increased patient compliance will save billions of dollars in healthcare costs each year by reducing hospital stays, doctor visits, and failures to follow prescriptions.[17]

\section{VII.CONCLUSION}

Microchip-based implanted drug delivery systems provide localised delivery by direct implantation of the device at the treatment site, as well as delivery on demand (pulsatile, adjustable continuous dose, and emergency administration, as well as a scheduled dosing cycle).

The medication delivery microchip is designed to allow for the safe, controlled release and storage of a variety of medications. This device is considerably more user-friendly and dependable than others that attempt to control the above-mentioned rate of drug release (electromechanical or systems made of polymers).The microchip is self-contained and can be produced using normal microfabrication methods, thus no patient or doctor contact is necessary. The device proposed can last over a year (assuming one dose per day); however, delivery capabilities are 
depending on patient demand.Internal drug delivery devices are being developed today leveraging the emerging disciplines of micro and nanotechnology to sense, excite, distribute to, and record from biological systems. Because some of these devices are programmable, medications may be stored and delivered on demand, either ahead of time or in real time.A silicon microchip that can regulate the ondemand release of one or more medications. When the anode membrane is dissolved by electrochemical disintegration, drugs in solid, liquid, or gel form might be stored in small reservoirs and released in controlled patterns. It's also feasible to make a biodegradable microchip that won't need to be removed in the future.

Implantable drug delivery technologies, such as microchips, would improve pharmaceutical safety, tolerability, and efficacy by permitting a more constant plasma drug level, similar to existing delayed-release antidepressants. These encouraging results point to the possibility of using microchipbased implant technology to deliver more therapeutic peptides and proteins.

They also show that drug delivery from a variety of reservoirs is not restricted to solution-phase drug formulations, and that stable solid-phase drug formulations may be packed and released in vivo. Microchips have enormous promise in medical diagnostics, microbiology, chemical detection, and industrial monitoring and control, to name a few. In the near future, the "microchip" will be used to dispense a variety of strong medicines.

\section{REFERENCES}

[1]. Bhowmik D, Gopinath H, Kumar BP, Duraivel S, Kumar KS. Microchip Drug Delivery-New Era Of Drug Delivery System. The Pharma Innovation. 2012 Dec 1;1(10, Part A):1.

[2]. Lee SH, Park M, Park CG, Lee JE, Prausnitz MR, Choy YB. Microchip for sustained drug delivery by diffusion through microchannels. Aaps Pharmscitech. 2012 Mar;13(1):211-7.

[3]. Sutradhar KB, Sumi CD. Implantable microchip: the futuristic controlled drug delivery system. Drug delivery. 2016 Jan 2;23(1):1-1.

[4]. Santini JT Jr, Richards AC, Scheidt RA, Cima MJ, Langer RS. Microchip technology in drug delivery. Ann Med. 2000 Sep;32(6):377-9.

[5]. Khirwadkar P, Sisodiya D, Dashora K. MICROCHIP TECHNOLOGY: A NEW APPROACH IN NOVEL DRUG DELIVERY SYSTEM. Int J of Biomed \&amp; Adv Res 2012 Apr. 1 [cited 2021 Jun. 26];3(3):149-61

[6]. Khirwadkar P, Singh D, Dashora K. Microchip technology: a new approach in novel drug delivery system. IJBAR. 2012;3(3):149-61

[7]. Vadlapatla R, Wong EY, Gayakwad SG. Electronic drug delivery systems: An overview. Journal of Drug Delivery Science and Technology. 2017 Oct 1;41:359-66.

[8]. Langer R. Drug delivery. Drugs on target. Science (New York, NY). 2001 Jul 1;293(5527):58-9.

[9]. Sutradhar KB, Sumi CD. Implantable microchip: the futuristic controlled drug delivery system. Drug delivery. 2016 Jan 2;23(1):1-1.

[10]. Santini Jr JT, Cima MJ, Langer RS, inventors; Massachusetts Institute of Technology, assignee. Microchip drug delivery devices. United States patent US 5,797,898. 1998 Aug 25.

[11]. Middelkamp HHT, van der Meer AD, Hummel JM, Stamatialis DF, Mummery CL, Passier R, et al. Organs-on-Chips in Drug Development: The Importance of Involving Stakeholders in Early Health Technology Assessment. Appl Vitr Toxicol 2016;2(2):74-81.

[12]. Jodat YA, Kang MG, Kiaee K, Kim GJ, Martinez $\mathrm{AFH}$, Rosenkranz A, et al. Human-Derived Organ-on-a-Chip for Personalized Drug Development. Curr Pharm Des 2019;24(45):5471-86. 
[13]. Cui P, Wang S. Application of micro fl uidic chip technology in pharmaceutical analysis : A review. J Pharm Anal 2019;9(4):238-47

[14]. Esch EW, Bahinski A, Huh D. Organs-on-chips at the frontiers of drug discovery. Nat Rev Drug Discov 2015;14(4):248-605.

[15]. Song JW, Cavnar SP, Walker AC, Luker KE, Gupta M, Luker GD, et al. Microfluidic Endothelium for Studying the Intravascular Adhesion of Metastatic Breast Cancer Cells. 2009;4(6).

[16]. Tyagi S, Tyagi R, Chamoli P, Negi N, Gupta A. Health Science Communication. 2020;13(3):1218-22

[17]. Garg A, Panday P, Patel V. Microchip: A ubiquitous technique for drug delivery. Asian Journal of Biomaterial Research. 2016;2(1):22-8

[18]. Eltorai AE, Fox H, McGurrin E, Guang S. Microchips in medicine: current and future applications. BioMed research international. 2016 Jun 7;2016.

[19]. Matheson R. Major step for implantable drugdelivery device.

[20]. https://www.fishersci.com/shop/products/fisher -scientific-histoprep-tissue-capsules-2/15182218

[21]. Behold the microchip in your body. (2018, December 2). The Asian Age. https:/www.asianage.com/discourse/021218/be hold-the-microchip-in-your-body.html

[22]. Davis, M. (2021, May 22). Do COVID-19 vaccines contain metals or microchip? Viral magnet test videos claim it's true. Science Times.

https://www.sciencetimes.com/articles/31319/2 0210522/covid-19-vaccines-contain-metalsmicrochip-viral-magnet-test-videos.htm

[23]. EHR microchips: The past, present, and future Healthcare tech. (2018, January 3). Clinician Today. https://cliniciantoday.com/ehrmicrochips-the-past-present-and-future/

[24]. Electropages. (n.d.). The dangers of microchip implants. Latest Electronics News on
Components and Electrical Engineering. https://www.electropages.com/blog/2020/08/da ngers-microchip-implants

[25]. Fact check: Do not believe this hoax about microchip implant in coronavirus vaccine. (2020, September 29). India Today. https://www.indiatoday.in/fact-

check/story/fact-check-do-not-believe-thishoax-about-microchip-implant-in-coronavirusvaccine-1726551-2020-09-29

[26]. FDA approves use of electronic chips in medications. (2012, August 1). Medical Xpress medical research advances and health news. https://medicalxpress.com/news/2012-08-fdaelectronic-chips-medications.html

[27]. The future of medicine: Medical microchips. (2017, August 21). IT Briefcase. https://www.itbriefcase.net/the-future-ofmedicine-medical-microchips

[28]. Link, D., \& TODAY, U. (2021, May 26). Fact check: Medical microchip developed by Columbia University has nothing to do with vaccines. USA TODAY. https://www.usatoday.com/story/news/factchec k/2021/05/26/fact-check-missing-context-postsnew-microchip-development/5232378001/

[29]. Medical | Microchip technology. (n.d.). Smart | Connected | Secure | Microchip Technology. https://www.microchip.com/enus/solutions/medical

[30]. Medicine of the future: New microchip technology could be used to track smart pills. (n.d.). California Institute of Technology. https://www.caltech.edu/about/news/medicinefuture-new-microchip-technology-could-beused-track-smart-pills-79601

[31]. The microchip at 50: Its medical triumphs to date. (n.d.). Laboratory News. https://www.labnews.co.uk/article/2027848/the _microchip_at_50_its_medical_triumphs_to_da te 
[32]. Microchips in medicine: Current and future applications. (2016, June 7). Publishing Open Access research journals \& papers | Hindawi. https:/www.hindawi.com/journals/bmri/2016/1 743472/

[33]. Microchips in medicine: Current and future applications. (2018, June 24). PrepperFortress. https://www.prepperfortress.com/microchipsin-medicine-current-and-future-applications/

[34]. Microchips in medicine: Current and future applications. (n.d.). PubMed.

[35]. Mlblevins. (2011, November 17). Advantages of nanotechnology in medicine. Science Struck. https://sciencestruck.com/nanotechnologyadvantages-in-medicine

[36]. PMC, E. (n.d.). Europe PMC. Europe PMC. https://europepmc.org/article/PMC/4914739

\section{Cite this article as :}

Ranajit Nath, Ratna Roy, Jisu Das, Kritideepa Nath, Priyanka Kurmi, Ambika Mandal, "The Futuristic Microchip Drug Delivery System - A New Identification Tool", International Journal of Scientific Research in Science and Technology (IJSRST), Online ISSN : 2395-602X, Print ISSN : 23956011, Volume 8 Issue 4, pp. 59-73, July-August 2021. Available at doi $\quad$ : https://doi.org/10.32628/IJSRST21849

Journal URL : https://ijsrst.com/IJSRST21849 